\title{
Effect of degree of reduction of graphene oxide on thermomechanical properties of polymer nanocomposites
}

\author{
Vinay Panwar and Kaushik Pal* \\ Department of Mechanical and Industrial Engineering, \\ Indian Institute of Technology Roorkee, Roorkee-247667, India \\ *Email:pl_kshk@yahoo.co.in
}

Now-a-days, extensive research has been focused to study the applicability of graphene based nanofillers as reinforcement in polymer matrices. Production of graphene through bottom-up approaches was found to be much costly due to low-yields. Reduction of graphene oxide has been noticed capable of resolving this problem. The efforts to find an optimum reduction technique for GO reveals good efficiency of deoxigination via hydrazine hydrate treatment and thermal annealing $[1,2]$.However, a close comparative analysis of these two techniques revealed that different oxygen containing groups are reduced in these two methods [2]. The electrical and mechanical properties of such polymer nanocomposites had been the key area of interest from last many years. the comparative effects of different types of graphene derivatives as reinforcement for such polymer nanocomposites lacks in this run. In this work, we have studied the influence of degree of reduction of graphene oxide on mechanical, thermal and rheological properties of Acrylonitrile-butadiene-styrene (ABS) composites prepared via melt-mixing technique. Three different types of composites have been prepared by reinforcing graphene oxide (GO), chemically reduced graphene oxide (rGO-1) and thermally reduced graphene oxide (rGO-2) to ABS matrix.

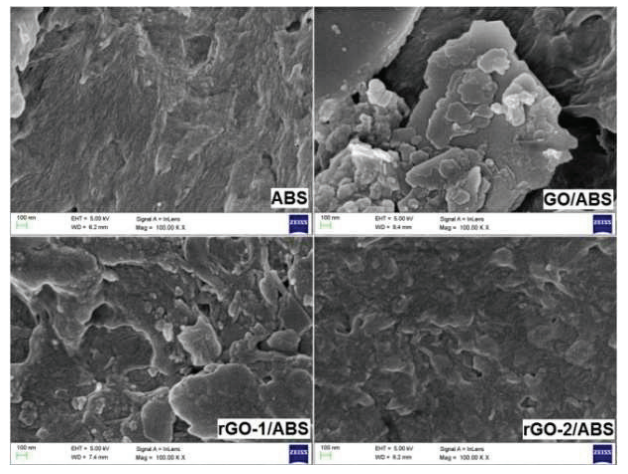

Figure 1: FESEM images of graphene-polymer nanocomposites

Morphological images of $\mathrm{GO} / \mathrm{rGO}$ reinforced composites are shown in Figure 4 depict that the fractured surface of pure ABS seems smooth representing the possibility of brittle fracture failure. GO reinforced composite shows clumpsing of GO nanosheets. This must be due to the presence of various functional groups on the side-walls and surface of nanosheets as well as strong van der Waals forces among the sheets. The dispersion seems to be improved with removal of functional groups through reduction of GO.

Figure 2: Tensile Strength of different composite specimen

The incorporation of rGO-1 resulted for significant improvement in tensile strength of the nanocomposite comparing to pure ABS. This direct towards improvement in mechanical properties with reduction of functional groups present in GO. Although heattreated $\mathrm{rGO}$ is having high degree of reduction but as reinforcement in ABS matrix, it resulted for diminishing tensile properties of the nanocomposite. Compared to rGO-1, rGO-2 shows massive decrease in tensile strength. These observations show poor network formation of $\mathrm{GO}$ and $\mathrm{rGO}-\mathrm{CT}$ in matrix, responsible for ineffective variation in mechanical properties of $\mathrm{GO} / \mathrm{ABS}$ and $\mathrm{rGO}-2 / \mathrm{ABS}$ nanocomposites.

We propose that chemically reduced graphene oxide acts better than other derivatives. Agglomeration of GO sheets due to presence of excessive functional groups in GO and poor interfacial bonding due to absence of functional groups in rGO-CT may be the reason for their deprived mechanical properties.

\section{References}

1. S. Pei, H.M. Cheng, Carbon 50 (2012) 3210.

2. X. Gao, J. Jang, S. Nagase, J. Phys. Chem. C114 (2010) 832 . 